\title{
Fate of Antenatally Diagnosed Pelvi-ureteric Junction Obstruction
}

\author{
Yogesh Kumar Sarin
}

Received: 23 June 2012 / Accepted: 30 November 2012 /Published online: 22 December 2012

(C) Dr. K C Chaudhuri Foundation 2012

To the Editor: I read article by Jain et al. [1] published in June 2012 issue of IJP with interest. I have the following reservations about the study design:

1. Did any of the patients in early pyeloplasty group have a non-obstructed renal scintigraphy curve at presentation? How many symptomatic patients in this subgroup had $>40 \%$ split renal function (SRF) with (a) obstructed renal curve, and (b) non-obstructed curve?

2. Although the authors mention under the heading 'inclusion criteria' that children having a follow-up of at least 24 mo or till their two consecutive renograms showed a non-obstructed pattern, either with or without operative correction, and later in follow up schedules that patients who underwent pyeloplasty had been followed up with physical examination, renal ultrasonography and scintigraphy at $3 \mathrm{mo}, 6 \mathrm{mo}$ and $1 \mathrm{y}$ following the pyeloplasty, Fig. 1 shows that the follow up in at least few of the postpyeloplasty patients in delayed pyeloplasty group was barely 3 mo.

3. The change in renal function was considered significant only if the change was $\geq 5 \%$. Whereas such small variations in SRF may be important in patients having their SRF hovering around $40-50 \%$, the specificity and sensitivity of similar small changes may not be any clinical significance when observed in patients with poorly functioning kidneys with SRF of $<25 \%$. For e.g., change of SRF from $12 \%$ to $15 \%$ is clinically inconsequential. Even the authors have conceded that a difference of $3.1 \%$ which is not more than standard error of renogram is inconsequential

Y. K. Sarin $(\bowtie)$

Department of Pediatrics Surgery,

Maulana Azad Medical College, Bahadur Shah Zafar Marg,

New Delhi 110002, India

e-mail: yksarin@hotmail.com
4. In Table 1, do the ANCOVA figures represent the mean renal function at last followup?

5. In discussion, it is mentioned that the mean renal function increased from $35.7 \%$ (range $12-50 \%$ ) to $40.6 \%$ (range 15-55\%) following pyeloplasty but never became $50 \%$ even though the surgery was performed at a mean age of $4.5 \mathrm{mo}$ (range $10 \mathrm{~d}-9.0 \mathrm{mo}$ ). This statement is self-contradictory.

6. The authors have not provided the $t-t / 2$ values for renal curves for any of the patients. This assumes importance as few patients had SRF as low as 12$15 \%$, and in these patients, it is very difficult to comment about the slope of renal curves and even $t-t$ $1 / 2$ values in such cases.

7. Did the authors mean 'kidney' when they state 'moiety' in Tables 1 and 2?

8. I wonder whether it is worthwhile to draw statistical differences between mean of subgroups when the sizes of the subgroups are so small and the SRF within each subgroup varied from $12-15 \%$ to $50-55 \%$.

9. The authors should have specified the cut-off times for delayed images when retention of tracer was studied in order to classify them as "maintained", "deteriorated" or "improved" drainage.

10. What happened to the patient in the 'No surgery group' who showed increase of dilatation of pelvis on follow up. I suspect that this patient also continued to have an obstructive pattern on renal scan. Why did authors choose not to operate on this patient?

\section{Reference}

1. Jain V, Agarwala S, Bhatnagar V, Gupta AK, Kumar R, Bal CS. Long term outcome of management of antenatally diagnosed pelviureteric junction obstruction. Indian J Pediatr. 2012;79:769-73. 